Original

\title{
Prevalencia de Incontinencia Urinaria y Vejiga Hiperactiva en la población española: Resultados del Estudio EPICC
}

\author{
Eduardo Martínez Agulló ${ }^{1}$, José L. Ruiz Cerdá ${ }^{1}$, Luis Gómez Pérez ${ }^{1}$, Miguel Ramírez Backhaus ${ }^{1}$, \\ Francisco Delgado Oliva ${ }^{1}$, Pablo Rebollo ${ }^{2}$, Diego González-Segura Alsina ${ }^{3}$, Daniel Arumi ${ }^{4}$, \\ Grupo de Estudio Cooperativo EPICC
}

${ }^{1}$ Servicio de Urología. Hospital Universitario La Fe, Valencia, ${ }^{2}$ BAP Health Outcomes Research, S.L. Oviedo,

${ }^{3}$ Departamento Médico, Laboratorios Almirall S.A. Barcelona, ${ }^{4}$ Departamento Médico, Pfizer S.A. Madrid.

\begin{abstract}
Resumen
Introducción: A pesar del creciente interés que despiertan en nuestro país la Incontinencia Urinaria (IU), la enuresis nocturna y la Vejiga Hiperactiva (VH), no existe ningún estudio epidemiológico que evalúe la prevalencia de estas patologías en los diferentes grupos sobre los que se asientan, de la población general de España.

Material y Métodos: Estudio epidemiológico, observacional, multicéntrico y de ámbito nacional. Se recogieron datos, a través de encuestas en 5 áreas representativas de todo el ámbito nacional y en cuatro grupos de la población: 1) Mujeres, laboralmente activas (entre 25 y 64 años); 2) Varones, laboralmente activos (entre 50 y 64 años); 3) Niños en enseñanza primaria (entre 6 y 11 años); y 4) Personas mayores de 65 años institucionalizadas con nivel cognitivo conservado. La encuesta a población adulta y ancianos incluía dos partes: 1) variables socio-demográficas y antecedentes de la historia clínica; y 2) sobre síntomas de VH e IU. La encuesta a niños incluía variables socio-demográficas y sobre ingesta de líquidos y control de la orina.

Resultados: El porcentaje de respuesta en los diferentes grupos del estudio osciló entre el 79,7\% y el 98\%. La prevalencia de $\mathrm{VH}$ y de IU aisladas en mujeres laboralmente activas fue de 2,69\% y 4,01\% respectivamente; en varones fue de 3,55\% y 0,56\%; en personas de más de 65 años fue de 9,14\% y 15,16\%. En conjunto el 9,94\% (95\% IC= 8,9-11,04) de las mujeres estudiadas padecían una o las dos patologías, porcentaje que fue de 5,14\% (95\% IC= 3,89 - 6,63) para varones y de $53,71 \%(95 \%$ IC $=50,56-56,85)$ para personas de más de 65 años. La prevalencia de enuresis nocturna en niños fue de $7,82 \%(95 \%$ IC= 6,62-9, 17).

Conclusión: La prevalencia de VH y/o IU en España se acerca al 10\% en mujeres entre 25 y 64 años, está alrededor del $5 \%$ en varones entre 50 y 65 años y es superior al 50\% en personas de más de 65 años de ambos sexos; la prevalencia de enuresis nocturna en niños entre 6 y 11 años está alrededor del 8\%.
\end{abstract}

Palabras clave: Incontinencia urinaria. Vejiga Hiperactiva. Epidemiología. Prevalencia. Población general.

\section{Prevalencia de Incontinencia Urinaria y Vejiga Hiperactiva en la población española: resultados del estudio EPICC}

Abstract

Background: Despite the growing interest on Urinary Incontinence (UI) in our country, nocturnal enuresis and Overactive Bladder $(\mathrm{OAB})$, there are no epidemiologic studies on the prevalence of these health problems in the different affected groups of the general population.

Patients and methods: This is an epidemiologic, observational, multicentre and national study. Data were collected by means of personal interviews in 5 representative areas from Spain and in 4 groups of population: 1) working women (2564 years old); 2) working men (50-64 years old); 3) children attending primary school (6-11 years old); and 4) elderly institutionalized subjects (over 65 years old) with no mental impairment. The Interview addressed to adult population included two parts: 1) socio-demographic variables and clinical history; and 2) data about OAB and UI symptoms. The Interview addressed to children included socio-demographic variables and questions about liquid intake and urine control.

Results: Percentage of answer to interview in the different groups varied between $79.7 \%$ and $98 \%$. Prevalence of isolate $\mathrm{OAB}$ and UI in working women were $2.69 \%$ and $4.01 \%$ respectively; in men were $3.55 \%$ and $0.56 \%$; in elderly were $9.14 \%$ and $15.16 \%$. In total, $9.94 \%(95 \% \mathrm{CI}=8.9-11.04)$ of the women under study suffer one or both health problems; this percentage was 5.14\% (95\% CI= 3.89-6.63) in men and 53.71\% (95\% CI= 50.56-56.85) in elderly. Prevalence of nocturnal enuresis in children was $7.82 \%(95 \% \mathrm{CI}=6.62-9.17)$.

Conclusions: Prevalence of OAB and/or UI in Spain is near 10\% of women between 25 and 64 years, is around 5\% in men between 50 and 64 years and it is over $50 \%$ in persons over 65 years; prevalence of nocturnal enuresis in children between 6 and 11 years is around $8 \%$.

Keywords: Urinary Incontinence. Overactive Bladder. Epidemiology. Prevalence. General population. 
T a incontinencia urinaria (IU) es la pérdida involunAtaria de orina objetivamente demostrable que origina un problema social o higiénico ${ }^{1}$. Según la nueva definición de la ICS, la IU es la manifestación por parte del paciente de escape de orina. Además, la IU debe ser descrita especificando los factores relevantes como el tipo, frecuencia, gravedad, factores desencadenantes, impacto social, efecto sobre la higiene y calidad de vida, medidas utilizadas para evitar los escapes de orina y si el individuo desea o no ayuda para tratar el problema de incontinencia. A pesar de que la incontinencia no implica un pronóstico de gravedad, limita la autonomía, reduce la autoestima y deteriora sensiblemente la calidad de vida de quien la padece $^{2}$. Por otro lado, la vejiga hiperactiva (VH) es definida por la presencia de urgencia, con o sin incontinencia urinaria de urgencia, a menudo acompañada de frecuencia y nocturia ${ }^{1}$. Su síntoma cardinal, la urgencia, ha sido relacionado con un gran impacto en la calidad de vida, superior al de los otros síntomas que pueden formar parte de la VH: incontinencia de urgencia, frecuencia y nocturia ${ }^{3}$. En ambas patologías, IU y VH, quienes las padecen con poca frecuencia acuden por ellas a los servicios sanitarios $^{2}$. Según un trabajo realizado en nuestro país ${ }^{4} \mathrm{el}$ $56,8 \%$ de las mujeres con IU estudiadas no habían visitado al médico por este problema y según otro estudio $^{5}$ este porcentaje sería superior al 70\%.

En el caso de la IU, la heterogeneidad de cifras de prevalencia es consecuencia de las diferencias entre los estudios respecto de la definición de incontinencia utilizada (en términos de números de escapes y su frecuencia), de las tasas de respuesta, la inclusión de sujetos institucionalizados, los métodos de recogida de datos, las preguntas usadas en los cuestionarios y la manera en que son respondidas, el periodo sobre el que se reporta la IU y la gravedad o importancia de la $\mathrm{IU}^{6}$. Con estos condicionantes no es de extrañar que se encuentren diferencias considerables entre los estudios realizados en un mismo país, como por ejemplo Estados Unidos, país en el que durante los últimos 5 años se han realizado al menos 12 estudios epidemiológicos de prevalencia de IU. En uno sólo de estos estudios, publicado muy recientemente ${ }^{7}$, se recogieron datos de ambos sexos y de tres grupos raciales (adultos entre 30 y 79 años) encontrando una prevalencia de sólo el 8\% (10,4\% en mujeres y 5,3\% en varones). Sin embargo otros dos estudios realizados en muestras de varones ${ }^{8,9}$ se encontraron cifras sensible- mente superiores: $12,7 \%$ y $17 \%$. También en los 9 estudios realizados en mujeres se encuentran cifras muy diferentes que oscilan entre el 15\% cuando se estudian mujeres de origen latino de más de 65 años $^{10}$ o el $18 \%$ en mujeres de origen asiático ${ }^{11}$, y hasta el $40 \%$ cuando se estudian mujeres no institucionalizadas ${ }^{12-17}$. En España también se encuentran importantes diferencias entre las cifras de prevalencia de IU de diferentes estudios, que en parte están también motivadas por la fecha de realización del estudio, en función a la publicación de la definición de la ICS antes mecionada ${ }^{1}$. Así en los estudios epidemiológicos publicados antes de $2003^{18}$, en personas de más de $60^{19}$ o de 65 años ${ }^{20}$ se obtienen cifras cercanas al 40\%, mientras que después de esa fecha las cifras son inferiores: $35,1 \%$ en sujetos de más de 64 años $^{21,22}, 23 \%$ en mujeres mayores de 18 años $^{5}, 20 \%$ en mujeres en edad laboral ${ }^{4}$ y $14 \%$ en mujeres entre 40 y 64 años ${ }^{22}$.

Respecto a la prevalencia de $\mathrm{VH}$, los estudios son más escasos y fundamentalmente se han realizado después de la publicación de la definición de la $\mathrm{ICS}^{1}$. En España únicamente se ha identificado un estudio reciente ${ }^{23}$ realizado en sujetos de más de 40 años, en el que se cifró la prevalencia en el 21,5\%, siendo mayor para mujeres $(25,6 \%)$ que para varones $(17,4 \%)$. Este estudio también evidenció que sólo el $28,4 \%$ de los sujetos con VH habían sido diagnosticados y sólo el 16,7\%, recibían tratamiento para la VH. Así, parece que el síndrome de VH, como la IU, está infra-diagnosticado e infra-tratado. Anteriormente a este trabajo, se habían publicado los resultados de un estudio epidemiológico ${ }^{24}$ realizado en 16.776 sujetos mayores de 40 años, de seis países europeos, que incluía datos de España. Según este estudio, la prevalencia de VH en nuestro país era del $22 \%$ y en el conjunto de los países del estudio, 16,6\%. Las cifras de prevalencia de otros estudios consultados varían entre el $11,8 \%$ de un estudio realizado en Canadá, Alemania, Italia, Suecia y Reino Unido ${ }^{25}$ y el 29,9\% de un estudio realizado en 11 países de Asia $^{26}$, pasando por el 12,4\% de Japón ${ }^{27}$, el 13,9\% y 18, 1\% de dos estudios en Canadá ${ }^{28,29}$, 16,9\% de Taiwan ${ }^{30}$, el 18,9\% de Brasil $^{31}$.

La enuresis nocturna es definida como cualquier pérdida involuntaria de orina durante el sueño, que ocurre más de una vez al mes ${ }^{1}$. Hasta donde conocemos no existe estudio alguno publicado con datos de España ${ }^{32}$. Sí se han publicado datos de otros paí- 
ses difícilmente extrapolables al nuestro. Así, un estudio realizado recientemente en Turquía ${ }^{33}$ cifró la prevalencia en el 20,8\% de una muestra de niños de escuela primaria; sin embargo en otros estudios realizados en países asiáticos, las prevalencias fueron mucho más bajas: en un estudio realizado en China $^{34}$ fue del $4,1 \%$, en Japón ${ }^{35}$ del 5,9\% y en Taiwan $^{36}$ del 6,8\%.

Sería preciso, por tanto, realizar un acercamiento correcto y objetivo a la vejiga hiperactiva y a la incontinencia urinaria, incluyendo la enuresis nocturna, y para ello se debería evaluar el grupo poblacional sobre el que asienta, intentando conocer a través de un amplio estudio epidemiológico. Así, se podrian conocer las bolsas patológicas que constituyen en los niños la enuresis nocturna, la incontinencia urinaria y vejiga hiperactiva en las mujeres pre y post-menopáusicas, la incontinencia urinaria y vejiga hiperactiva en el hombre y la incontinencia urinaria y vejiga hiperactiva en personas mayores de sesenta y cinco años institucionalizadas.

El objetivo del presente estudio institucional de la Asociación Española de Urología, fue detectar signos y síntomas de IU, enuresis nocturna y $\mathrm{VH}$, siguiendo las definiciones de la ICS, en la población general para conocer la prevalencia de estas patologías en España.

\section{SUJETOS Y MÉTODO}

Estudio epidemiológico, observacional, multicéntrico y de ámbito nacional en el que se recogió información mediante encuestas a personas que aceptaron participar en el proyecto después de ser informadas sobre los objetivos del mismo. Se recogieron por separado datos de cuatro grupos de la población: 1) Mujeres, laboralmente activas, con edades comprendidas entre 25 y 64 años; 2) Varones, laboralmente activos, con edades comprendidas entre 50 y 64 años; 3) niños de edades comprendidas entre 6 y 11 años en ciclo de enseñanza primaria; y 4) Personas mayores de 65 años institucionalizadas con nivel cognitivo conservado.

El tamaño muestral se calculó para cada uno de estos grupos de tal modo que, con un 95\% de seguridad, proporcionara una estimación de la prevalencia de incontinencia urinaria cuyo intervalo de confianza no fuera superior a un $\pm 1 \%$, en los grupos de mujeres y varones laboralmente activos y no superara el $\pm 3 \%$ en los grupos de niños y mayores de 65 años institucionalizados. Se consideró que la preva- lencia global conjunta de incontinencia urinaria en el grupo de mujeres de 25-65 y de varones de 50-65 no superaría un $12 \%$. Así, fijando un error $\alpha=0,05$ y un intervalo de confianza de un $\pm 1 \%$ serían necesarias 4.000 personas para cumplir dicho objetivo: 3.000 mujeres y 1.000 hombres. En los grupos de niños y mayores de 65 años institucionalizados, con una seguridad de un 95\% y un intervalo de confianza de un $\pm 3 \%$, en la más conservadora de las situaciones $(\mathrm{p}=\mathrm{q}=0,5)$, serían necesarias 1.000 personas en cada grupo.

Los datos se recogieron mediante una encuesta presencial, llevada a cabo por personal sanitario formado para tal fin. La encuesta a población adulta, varones entre 50 y 64 años y mujeres entre 25 y 64 años, se realizó en 5 áreas representativas de todo el ámbito nacional: Madrid, Barcelona, Valencia, Sevilla y Zaragoza, cada una bajo la supervisión de responsables médicos del Grupo de Estudio Cooperativo EPICC. La encuesta a niños se realizó en colegios ubicados en una provincia española representativa de todo el ámbito nacional (Alicante). La encuesta a personas mayores de 65 años se realizó en residencias ubicadas en una provincia española representativa de todo el ámbito nacional (Valencia).

La encuesta a población adulta y ancianos institucionalizados incluía dos partes: una parte de variables socio-demográficas (edad, sexo, peso, talla, estado civil, nivel de estudios, actividad profesional y situación laboral) y antecedentes de la historia clínica (presencia de trastornos de próstata en varones y número de hijos y otros datos de historia ginecológica en mujeres) y otra sobre síntomas de vejiga hiperactiva e incontinencia urinaria. Esta segunda parte incluía una serie de preguntas enfocadas a obtener información sobre la presencia de Vejiga Hiperactiva (VH), Incontinencia Urinaria (IU), Frecuencia, nocturia y enuresis nocturna.

La encuesta a niños incluía variables sociodemográficas (edad, sexo, peso, talla, curso escolar y situación de convivencia) y otras sobre ingesta de líquidos y sobre el control de la orina.

Se consideró que un sujeto tenía IU en los casos en los que en el último año se le había escapado la orina en más de una ocasión y la frecuencia con que se producían las pérdidas era de al menos 3 veces al año. Se consideró que un sujeto tenía $\mathrm{VH}$ en los casos en los que referían sensación repentina e imperiosa de ganas de orinar y con una frecuencia 
con qué se producen los deseos incontrolables de orinar de al menos 3 veces al año. El síntoma frecuencia estaba definido para la realización de más de 8 micciones diarias, el síntoma nocturia por más de 2 micciones en una noche, y la presencia de enuresis nocturna se consideró cuando los episodios de pérdida de orina durante el sueño se producían más de 1 vez al mes.

\section{ANÁLISIS ESTADÍSTICO}

Para el proceso de los datos se aplicaron los procedimientos operativos adecuados que garantizan su calidad, como la doble entrada de datos por personal independiente y la generación de filtros de consistencia. Se realizó una depuración lógica de datos. Todo ello se realizó con programas de gestión de datos validados por las agencias reguladoras (Oracle ${ }^{\circledR}$ Clinical) y los análisis estadísticos y tablas se generarán mediante el programa SAS versión 9.

Se calcularon, de todas las variables, los parámetros descriptivos: media, desviación estándar y tamaño en las cuantitativas que se ajustaron a Gauss (test de Shapiro-Wilk) y mediana y rango intercuartílico en las que no se ajustaron a la curva normal. Las varia- bles cualitativas se expresaron mediante las frecuencias relativas porcentuales. Respecto a la variable principal (prevalencia) se calculó el intervalo de confianza para un 95\% de seguridad.

\section{RESULTADOS}

El porcentaje de respuesta de los sujetos a los que se les solicitó participar en los diferentes grupos del estudio fue 85,83\% (3.090/3.600) en mujeres entre 25 y 64 años, 89\% (1.071/1.200) en varones entre 50 y 64 años, 79,72\% (1.345/1.687) en niños escolarizados en enseñanza primaria y $98 \%$ (1021/1042) en ancianos institucionalizados. De estos no se consideraron válidos para el análisis 66 cuestionarios de niños y 25 de ancianos institucionalizados. Así las muestras sometidas a análisis estadístico fueron: 3.090 mujeres entre 25 y 64 años, 1.071 varones entre 50 y 64 años, 1.279 niños $(75,82 \%)$ y 996 ancianos $(97,6 \%)$

Las características principales de las diferentes muestras, de mujeres entre 25 y 64 años, de varones entre 50 y 65 años, de niños entre 6 y 11 años y de personas de más de 65 años institucionalizados, se presentan respectivamente en las Tablas 1 a 4 .

Tabla 1. Descripción de la muestra de mujeres entre 25 y 64 años (N=3.090)

\begin{tabular}{|c|c|c|c|c|}
\hline Edad media (D.E.) & \multicolumn{2}{|c|}{$43,39(11,32)$} & \multicolumn{2}{|c|}{ I.C. 95\% $(42,99-43,79)$} \\
\hline IMC medio (D.E.) & \multicolumn{2}{|c|}{$24,42(4,20)$} & \multicolumn{2}{|c|}{ I.C. $95 \%(24,27-24,57)$} \\
\hline Estado civil \% & $\begin{array}{c}\text { Soltera } \\
25,7\end{array}$ & $\begin{array}{c}\text { Casada } \\
64,1\end{array}$ & $\begin{array}{c}\text { Viuda } \\
3,3\end{array}$ & $\begin{array}{c}\text { Divorciada } \\
5,7\end{array}$ \\
\hline Nivel estudios \% & $\begin{array}{c}\text { Sin estudios } \\
1,7\end{array}$ & $\begin{array}{c}\text { Primarios } \\
28,2\end{array}$ & $\begin{array}{c}\text { Secundarios } \\
31,6\end{array}$ & $\begin{array}{l}\text { Universitarios } \\
\quad 37,3\end{array}$ \\
\hline Actividad profesional \% & $\begin{array}{c}\text { Ama de casa } \\
17,4\end{array}$ & $\begin{array}{c}\text { Prof. Liberal } \\
5,7\end{array}$ & $\begin{array}{l}\text { Empleado } \\
\quad 63,5\end{array}$ & $\begin{array}{c}\text { Obrero } \\
1,6\end{array}$ \\
\hline Número de hijos medio (D.E.) & \multicolumn{2}{|c|}{$1,29(1,22)$} & \multicolumn{2}{|c|}{ I.C. $\mathbf{9 5 \%}(1,24-1,33)$} \\
\hline Menopausia \% & \multicolumn{2}{|c|}{32,5} & \multicolumn{2}{|c|}{$\begin{array}{l}\text { Edad media de menopausia (D.E.) } \\
\qquad 49(4,2) \text { años }\end{array}$} \\
\hline Prolapso vaginal \% & \multicolumn{2}{|c|}{3,4} & & \\
\hline Tratamiento hormonal \% & \multicolumn{2}{|c|}{5,8} & & \\
\hline
\end{tabular}

Tabla 2. Descripción de la muestra de varones entre 50 y 65 años $(\mathrm{N}=1.071)$

\begin{tabular}{|c|c|c|c|c|c|}
\hline Edad media (D.E.) & \multicolumn{2}{|c|}{$55,94(4,35)$} & \multicolumn{3}{|c|}{ I.C. 95\% $(55,67-56,20)$} \\
\hline Estado civil \% & $\begin{array}{c}\text { Soltero } \\
6,9\end{array}$ & $\begin{array}{c}\text { Casado } \\
84,5\end{array}$ & \multicolumn{2}{|c|}{$\begin{array}{l}\text { Viudo } \\
2,4\end{array}$} & $\begin{array}{c}\text { Divorciado } \\
5,7\end{array}$ \\
\hline Nivel estudios \% & $\begin{array}{c}\text { Sin estudios } \\
4,4\end{array}$ & $\begin{array}{c}\text { Primarios } \\
47,7\end{array}$ & \multicolumn{2}{|c|}{$\begin{array}{c}\text { Secundarios } \\
21,7\end{array}$} & $\begin{array}{c}\text { Universitarios } \\
\quad 24,2\end{array}$ \\
\hline Actividad profesional \% & $\begin{array}{c}\text { Amo de casa } \\
0,19\end{array}$ & $\begin{array}{c}\text { Prof. Liberal } \\
9,7\end{array}$ & $\begin{array}{c}\text { Directivo } \\
13,8\end{array}$ & $\begin{array}{c}\text { Empleado } \\
52,9\end{array}$ & $\begin{array}{c}\text { Obrero } \\
17,9\end{array}$ \\
\hline Trastornos de próstata \% & & & $\begin{array}{l}\mathrm{HBP} \\
5,0\end{array}$ & $\begin{array}{c}\text { Prostatitis } \\
3,9\end{array}$ & $\begin{array}{c}\text { Cáncer } \\
0,5\end{array}$ \\
\hline
\end{tabular}


Tabla 3. Descripción de la muestra de niños entre 6 y 11 años $(\mathrm{N}=1.279)$.

\begin{tabular}{|c|c|c|c|c|c|c|}
\hline Edad media (D.E.) & $8,4(1,7)$ & & & & & \\
\hline Sexo varón $\mathbf{n}(\%)$ & $647(50,6)$ & & & & & \\
\hline Curso escolar $\mathbf{n}(\%)$ & $\begin{array}{l}\text { Primero } \\
227(17,7)\end{array}$ & $\begin{array}{l}\text { Segundo } \\
221(17,3)\end{array}$ & $\begin{array}{c}\text { Tercero } \\
221(17,3)\end{array}$ & $\begin{array}{c}\text { Cuarto } \\
234(18,3)\end{array}$ & $\begin{array}{l}\text { Quinto } \\
176(13,8)\end{array}$ & $\begin{array}{c}\text { Sexto } \\
200(15,6)\end{array}$ \\
\hline Viven con los padres $\%$ & & 95,4 & & & & \\
\hline $\mathrm{N}^{\circ}$ medio de vasos de líquido (D.E.) & $\begin{array}{c}\text { Desayuno } \\
1,09(0,4)\end{array}$ & $\begin{array}{c}\text { Entre } \\
1,34(0,8)\end{array}$ & $\begin{array}{c}\text { Comida } \\
1,57(0,6)\end{array}$ & $\begin{array}{l}\text { Entre } \\
1,63(1)\end{array}$ & $\begin{array}{c}\text { Cena } \\
1,41(0,6)\end{array}$ & $\begin{array}{l}\text { Acostarse } \\
0,89(0,5)\end{array}$ \\
\hline $\begin{array}{l}\text { Aguantan las ganas de orinar } \\
\text { durante el dia } \%\end{array}$ & & 95,8 & & \multicolumn{3}{|c|}{$\begin{array}{l}\mathrm{N}^{\mathrm{o}} \text { medio de horas (D.E.) } \\
\qquad 3,68(1,7)\end{array}$} \\
\hline
\end{tabular}

Tabla 4. Descripción de la muestra de personas mayores de 65 años institucionalizadas (N=996)

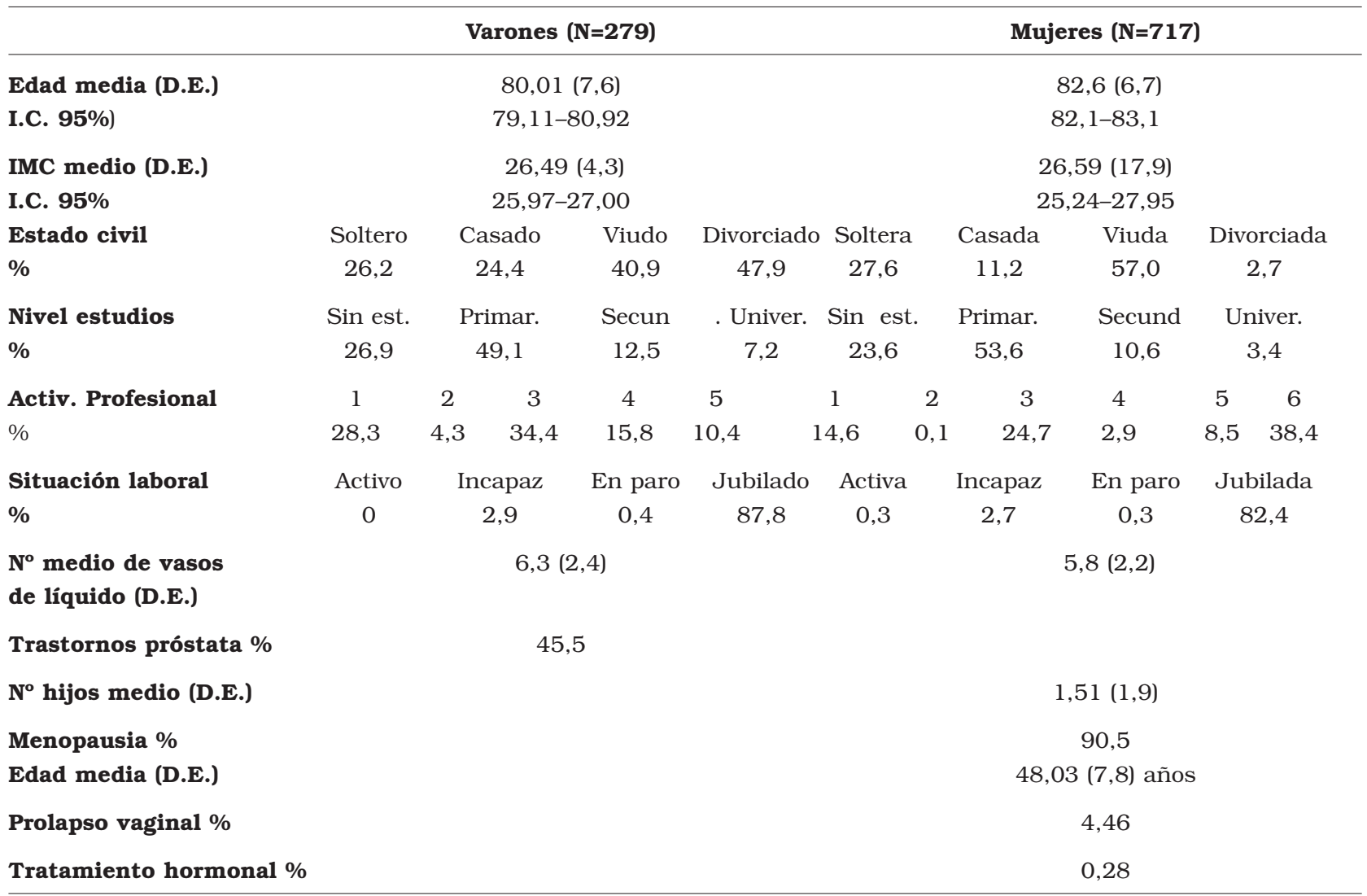

Actividad profesional: (1) profesión liberal; (2) directivo; (3) empleado; (4) obrero; (5) sin profesión; (6) ama de casa

La prevalencia de $\mathrm{VH}$ y de IU aisladas en mujeres entre 25 y 64 años fue respectivamente de 2,69\% y 4,01\%; además ambas patologías se presentaron juntas en el 3,24\% de las mujeres. En conjunto el 9,94\% (95\% IC= 8,9 - 11,04) de las mujeres entre 25 y 64 años padecían una o las dos patologías. Los resultados detallados para los diferentes grupos de edad se presentan en la Figura 1. La prevalencia de ambas patologías es claramente superior en el grupo de mayor edad. También puede observarse que en las mujeres de este rango de edad es más fre- cuente la presencia de IU que de $\mathrm{VH}$, patologías que se presentan juntas en el 2,06\% de las mujeres menores de 45 años, en el 2,57\% de las mujeres entre 45 y 54 años y en el 7,17\% de las mujeres entre 55 y 64 años.

La prevalencia de VH y de IU aisladas, en varones entre 50 y 65 años fue respectivamente de $3,55 \%$ y $0,56 \%$; además ambas patologías se presentaron juntas en el 1,03\% de los varones estudiados. En conjunto el 5,14\% (95\% IC= 3,89-6,63) de los varones padecían una o las dos patologías. Los 
resultados detallados para los diferentes grupos de edad se muestran en la Figura 2. Como puede verse en la Figura, la prevalencia de ambas patologías es casi el doble en el grupo de edad comprendida entre 55 y 64 años. En el conjunto de los varones estudiados es más frecuente la existencia de VH que de IU.

Respecto a la enuresis nocturna estudiada entre niños de 6 y 11 años, la prevalencia resultó ser de $7,82 \%(95 \% \mathrm{IC}=6,62-9,17)$. Los resultados detallados por sexo y edad se presentan en la Figura 3. La prevalencia es algo más del doble en niños respecto a niñas $(\mathrm{p}<0,0001)$ y disminuye claramente con la edad.

Por último la prevalencia de $\mathrm{VH}$ y de IU en personas de más de 65 años institucionalizadas fue de $9,14 \%$ y 15,16\% respectivamente; además ambas patologías se presentaron juntas en el 29,42\% de los sujetos estudiados. En conjunto el 53,71\% (95\% $\mathrm{IC}=50,56-56,85)$ de las personas de más de 65

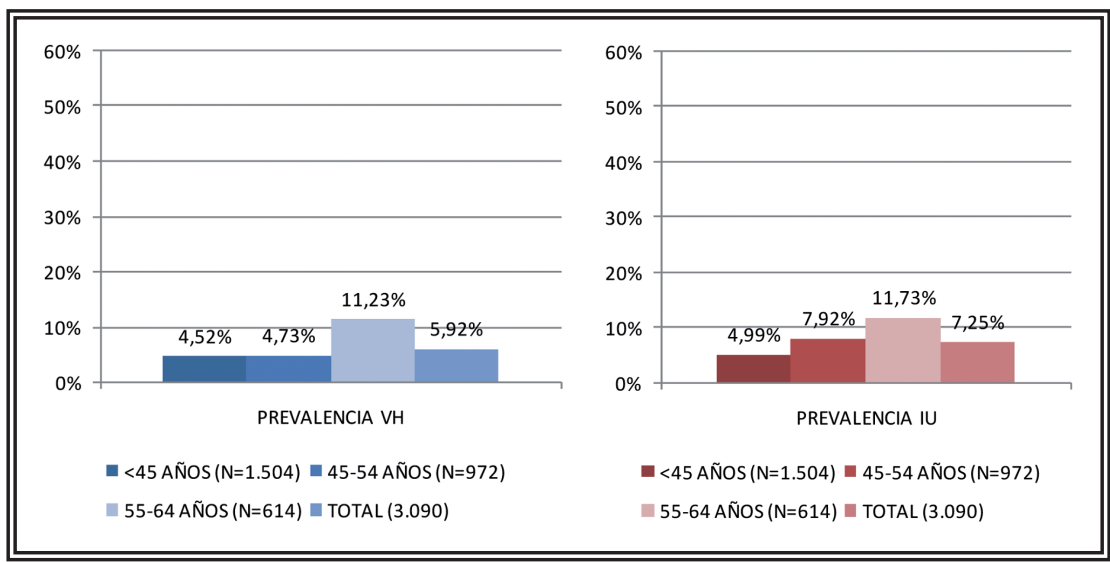

FIGURA 1. Prevalencia de Vejiga Hiperactiva y de Incontinencia Urinaria en la muestra de mujeres entre 25 y 64 años ( $N=3.090)$.

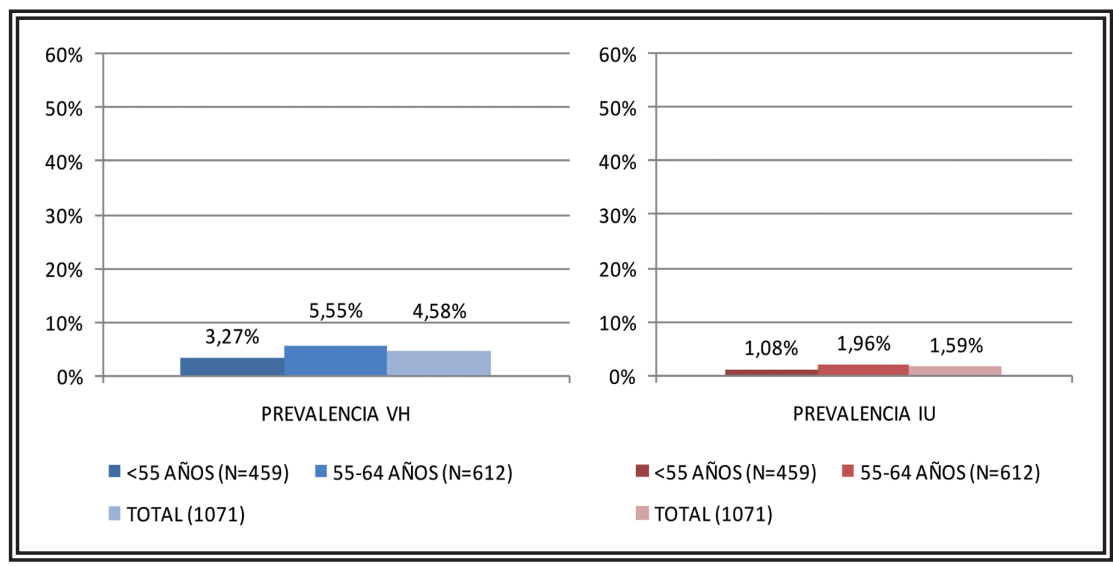

FIGURA 2. Prevalencia de Vejiga Hiperactiva y de Incontinencia Urinaria en la muestra de varones entre 50 y 65 años ( $N=1.071)$. años padecían una o las dos patologías. Los resultados detallados para ambos sexos se presentan en la Figura 4. En este rango de edad, la IU se presenta con mayor frecuencia en mujeres y la VH en varones. También puede observarse que la prevalencia global de IU es mayor que la de VH.

\section{DISCUSIÓN}

El estudio EPICC, proyecto Institucional de Asociación Española de Urología, es el primer estudio que proporciona datos epidemiológicos españoles fidedignos para la prevalencia de VH, de IU y de enuresis nocturna, a través del estudio de diferentes grupos de la población general: mujeres entre 25 y 64 años, varones entre 50 y 64 años, ancianos institucionalizados de ambos sexos y niños de ambos sexos escolarizados en enseñanza primaria. Según los resultados presentados, la prevalencia de $\mathrm{VH}$ y/o IU en España se acerca al 10\% en mujeres entre 25 y 64 años, está alrededor del 5\% en varones entre 50 y 65 años y es superior al $50 \%$ en personas de más de 65 años de ambos sexos; la prevalencia de enuresis nocturna en niños entre 6 y 11 años está alrededor del $8 \%$.

Respecto a la IU, la cifra de prevalencia en mujeres entre 25 y 64 años del presente estudio es sensiblemente inferior a las descritas en algunos estudios previos en nuestro país ${ }^{4,18,21,22}$ que oscilaron entre el $14 \%$ y el $40,6 \%$, y a las de estudios realizados en otros países como Estados Unidos ${ }^{11-17}$, que oscilan entre el 15 y el 40\%, excepto el estudio recientemente publicado antes citado ${ }^{7}$ que, aunque también es superior, se aproxima algo más a la del presente estudio: 10,4\%. En varones entre 50 y 64 años, la prevalencia de IU fue muy inferior a la encontrada en las mujeres de similar edad (ver figuras 1 y 2) y también inferior a la reportada en estudios previos realizados fuera de nuestro país ${ }^{7-9}$ (no se tiene constancia de estudios en muestras similares en nuestro país). Estas diferencias en las 


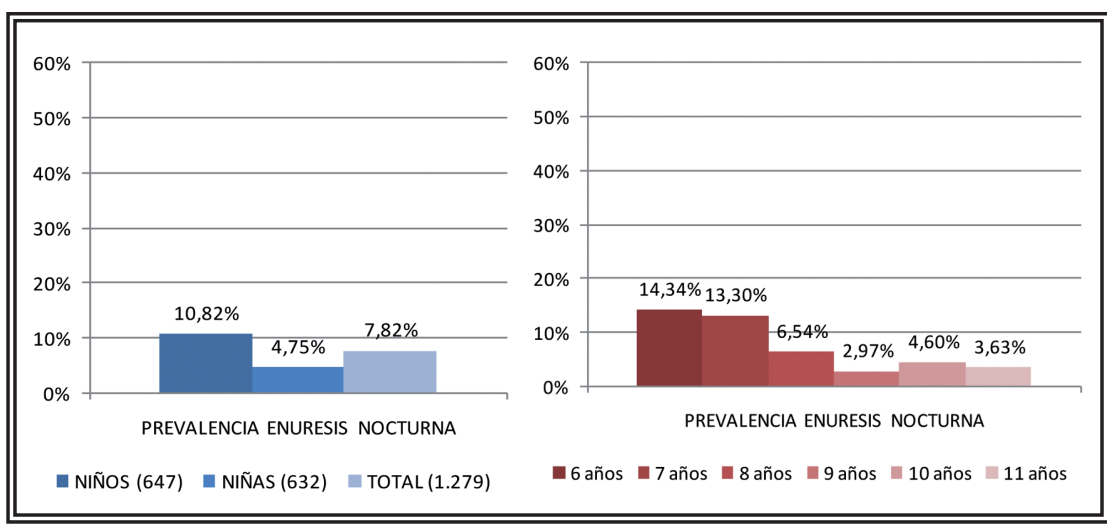

FIGURA 3. Prevalencia de Enuresis Nocturna en la muestra de niños entre 6 y 11 años ( $N=1.279)$.

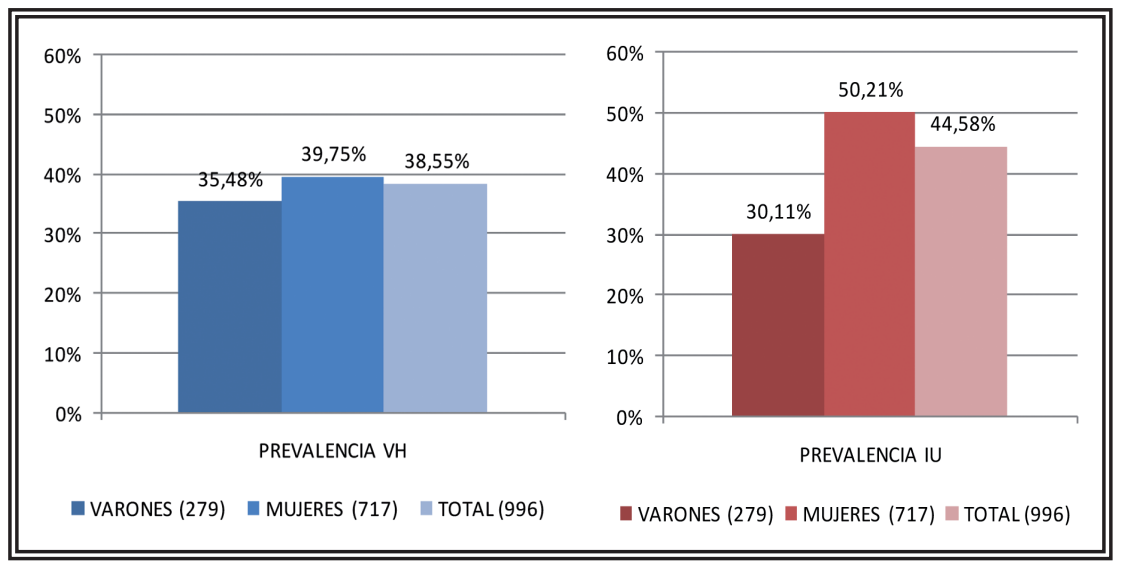

FIGURA 4. Prevalencia de Vejiga Hiperactiva y de Incontinencia Urinaria en la muestra de personas de más de 65 años institucionalizadas ( $N=996)$.

la identificación de VH. Así, si unimos a la cifra anterior, la de las mujeres con VH de más de 65 años institucionalizadas $(39,75 \%)$, probablemente las cifras resultantes serían más similares. También la prevalencia de $\mathrm{VH}$ en varones entre 50 y 64 años $(4,58 \%)$ es muy inferior a la previamente reportada en el estudio previo realizado en España ${ }^{23}$ que fue tan sólo del 17,4\%. Respecto a la prevalencia de VH en personas de más de 65 años institucionalizadas (38,55\%), no se han identificado estudios previos con muestras similares, con los que comparar los resultados obtenidos.

Respecto a la enuresis nocturna estudiada entre niños de 6 y 11 años, la prevalencia en el presente estudio $(7,82 \%)$ es claramente inferior a la de otros estudios previos realizados en España ${ }^{32}$ y Turquía ${ }^{33}$, aunque superior a las de los estudios de China ${ }^{34}$ y Japón ${ }^{35}$, similar a la del estudio de Taiwan ${ }^{36}$.

\section{Agradecimientos}

Agradecemos la colaboración de todos los médicos que han participa-

cifras de prevalencia respecto de las de otros estudios pueden ser debidas a que en el presente estudio se ha separado la VH de la IU, a que las muestras tenían diferente edad media y a que se han empleado criterios más exigentes para definir la IU (definición de IU de la ICS). Respecto a la prevalencia de IU en personas institucionalizadas de más de 65 años son similares a las de estudios previos realizados en nuestro país que la cifraron en alrededor del 40\% ${ }^{19-22}$ para muestras de sujetos residentes en la comunidad.

La prevalencia de $\mathrm{VH}$ en mujeres entre 25 y 64 años del presente estudio (5,92\%) es claramente inferior a la reportada en un estudio previo realizado en nuestro país que la cifró en $25,6 \%$ para mujeres de más de 40 años ${ }^{23}$. También es inferior a la prevalencia reportada en estudios previos realizados en otros países $^{24-31}$. Vuelve a intuirse una posible explicación a estas diferencias en la diferente edad de las mujeres incluidas y a los diferentes criterios aplicados para do en el estudio EPICC, así como la colaboración de Laboratorios Almirall S. A. y de Pfizer S.A. por facilitarnos la infraestructura necesaria para que el estudio EPICC pudiese llevarse a cabo.

\section{Grupo de Estudio Cooperativo EPICC}

P. Arañó Bertrán, S. Arlandis Guzmán, S. Armengol, D. Castro Díaz, J Conejero Sugrañes, I. Expósito, M. Giménez-Cidre, JM. Pena Outeriño, C. Rioja Sanz.

\section{REFERENCIAS}

1. Martínez Agulló E. Terminología de la función del tracto urinario inferior. Actas Urol Esp. 2005 ;29(1):5-7

2. Espuña PM. [Urinary incontinence in women]. Med Clin (Barc). 2003 Apr 5;120(12):464-472.

3. Coyne KS, Payne C, Bhattacharyya SK, Revicki DA, Thompson C, Corey R, Hunt TL. The impact of urinary urgency and frequency on health-related quality of life in overactive bladder: results from a national community survey. Value Health. 2004;7(4):455-463.

4. Modroño Freire MJ, Sánchez Cougil MJ, Gayoso Diz P, Valero Paternain M, Blanco Ramos M, Cuña Ramos FO. [Study of the prevalence of urinary incontinence in women from 18 to 65 and its influence on their quality of life]. Aten Primaria. 2004;34(3):134139. 
5. Hunskaar S, Lose G, Sykes D, Voss S. The prevalence of urinary incontinence in women in four European countries. BJU Int. 2004;93(3):324-230.

6. Botlero R, Urquhart DM, Davis SR, Bell RJ. Prevalence and incidence of urinary incontinence in women: review of the literature and investigation of methodological issues. Int $\mathrm{J}$ Urol. 2008; 15 (3):230-234.

7. Tennstedt SL, Link CL, Steers WD, McKinlay JB. Prevalence of and risk factors for urine leakage in a racially and ethnically diverse population of adults: the Boston Area Community Health (BACH). Am J Epidemiol. 2008. 15;167(4):390-399.

8. Diokno AC, Estanol MV, Ibrahim IA, Balasubramaniam M. Prevalence of urinary incontinence in community dwelling men: a cross sectional nationwide epidemiological survey. Int Urol Nephrol. 2007;39(1):129-136.

9. Anger JT, Saigal CS, Litwin MS; Urologic Diseases of America Project, The prevalence of urinary incontinence among community dwelling men: results from the National Health and Nutrition Examination survey. J Urol. 2006 Feb;175(2):601-614.

10. Espino DV, Palmer RF, Miles TP, Mouton CP, Lichtenstein MJ, Markides KP. Prevalence and severity of urinary incontinence in elderly Mexican-American women. $\mathrm{J}$ Am Geriatr Soc. 2003;51(11):1580-1586.

11. Huang AJ, Thom DH, Kanaya AM, Wassel-Fyr CL, Van den Eeden SK, Ragins AI, Subak LL, Brown JS. Urinary incontinence and pelvic floor dysfunction in Asian-American women Am J Obstet Gynecol. 2006 Nov;195(5):1331-1337 .

12. Minassian VA, Stewart WF, Wood GC. Urinary incontinence in women: variation in prevalence estimates and risk factors. Obstet Gynecol. 2008 ;111(2 Pt 1):324-331.

13. Dooley Y, Kenton K, Cao G, Luke A, Durazo-Arvizu R, Kramer $\mathrm{H}$, Brubaker L. Urinary incontinence prevalence: results from the National Health and Nutrition Examination Survey. J Urol. 2008;179(2):656-561. .

14. Kinchen KS, Lee J, Fireman B, Hunkeler E, Nehemiah JL, Curtice TG. The prevalence, burden, and treatment of urinary incontinence among women in a managed care plan $\mathrm{J}$ Womens Health (Larchmt). 2007;16(3):415-422.

15. Anger JT, Saigal CS, Litwin MS; Urologic Diseases of America Project. The prevalence of urinary incontinence among community dwelling adult women: results from the National Health and Nutrition Examination Survey. J Urol. 2006;175(2):601614.

16. Fultz N, Girts T, Kinchen K, Nygaard I, Pohl G, Sternfeld B.P revalence, management and impact of urinary incontinence in the workplace. Occup Med (Lond). 2005;55(7):552-557.

17. Melville JL, Katon W, Delaney K, Newton K. Urinary incontinence in US women: a population-based study. Arch Intern Med. 2005;14;165(5):537-542.

18. Bretones J, Pino M, García M, Fajardo M, Sáez J, Ortega, I.: Incontinencia urinaria en mujeres en edad adulta: estudio descriptivo en una poblacion rural. Aten Primaria1997, 20: 45,

19. Sánchez, R., Rupérez, O., Delgado, M., Fernández, R., and Hernando, M.: Prevalencia de incontinencia urinaria en la población mayor de 60 años atendida en atención primaria. Aten Primaria, 24: 421, 1999. .

20. Gavira, F., Caridad, J., Pérez del Molino, J., Valderrama, E., López, M., Romero, M. et al.: Uso de accesorios para incontinencia urinaria en los ancianos de la Zona Basica de Salud de Cabra (Cordoba). Aten Primaria, 25: 556, 2000. .

21. Rodríguez-Lama M, Martínez-Calvo JR, Rodríguez-Pérez C, Melero-Brezo M, García-Cepeda JR. Prevalencia de la incontinencia urinaria en personas mayores de 64 años en Galicia. Gac Sanit. 2003 Sep-Oct;17(5):409-411.
22. Nieto B. E., Camacho, P. J., Davila, A., V, Ledo Garcia, M. P., Moriano, B. P., Perez, L. M. et al.: [Epidemiology and impact of urinary incontinence in women between 40 and 65 in a health area of Madrid]. Aten Primaria, 32: 410, 2003.

23. Castro D, Espuña M, Prieto M, Badia, X.. Prevalencia de la vejiga hiperactiva eb España. Estudio basado en una oblación. Arch Esp Urol. 2005 Mar;58(2):131-138.

24. Milsom I, Abrams P, Cardozo L, Roberts RG, Thüroff J, Wein AJ. How widespread are the symptoms of an overactive bladder and how are they managed? A population-based prevalence study. BJU Int. 2001;87(9):760-766.

25. Irwin DE, Milsom I, Hunskaar S, Reilly K, Kopp Z, Herschorn S, Coyne K, Kelleher C, Hampel C, Artibani W, Abrams P. Population-based survey of urinary incontinence, overactive bladder, and other lower urinary tract symptoms in five countries: results of the EPIC study. Eur Urol. 2006;50(6):1306131.

26. Moorthy P, Lapitan MC, Quek PL, Lim PH. Prevalence of overactive bladder in Asian men: an epidemiological survey. BJU Int. 2004;93(4):528-351.

27. Homma Y, Yamaguchi O, Hayashi K. Neurogenic Bladder Society Committee. An epidemiological survey of overactive bladder symptoms in Japan. BJU Int. 2005;96(9):1314-1348.

28. Herschorn S, Gajewski J, Schulz J, Corcos J. A populationbased study of urinary symptoms and incontinence: the Canadian Urinary Bladder Survey. BJU Int. 2008;101(1):5258.

29. Corcos J, Schick E. Prevalence of overactive bladder and incontinence in Canada. Can J Urol, 2004;11(3):2278-2284.

30. Yu HJ, Liu CY, Lee KL, Lee WC, Chen TH. Overactive bladder syndrome among community-dwelling adults in Taiwan: prevalence, correlates, perception, and treatment seeking. Urol Int, 2006;77(4):327-333.

31. Teloken C, Caraver F, Weber FA, Teloken PE, Moraes JF, Sogari PR, Graziottin TM. Overactive bladder: prevalence and implications in Brazil. Eur Urol. 2006 ;49(6):1087-1092.

32. SINUG; Grupo Español de Urodinámica Propuestas de adaptación terminológica al español de la estandarización de la terminología del tracto urinario inferior en niños y adolescentes de la ICCS. Actas Urol Esp. 2008 Apr;32(4):371-389.

33. Carman KB, Ceran O, Kaya C, Nuhoglu C, Karaman MI. Nocturnal enuresis in Turkey: prevalence and accompanying factors in different socioeconomic environments. Urol Int. 2008; 80(4):362-366.

34. Wen JG, Wang QW, Chen Y, Wen JJ, Liu K. An epidemiological study of primary nocturnal enuresis in Chinese children and adolescents. Eur Urol. 2006;49(6):1107-1113.

35. Kajiwara M, Inoue K, Kato M, Usui A, Kurihara M, Usui T. Nocturnal enuresis and overactive bladder in children: an epidemiological study. Int J Urol. 2006;13(1):36-41.

36. Tai HL, Chang YJ, Chang SC, Chen GD, Chang CP, Chou MC. The epidemiology and factors associated with nocturnal enuresis and its severity in primary school children in Taiwan. Acta Paediatr. 2007;96(2):242-245.

Correspondencia autor: Dr. Pablo Rebollo

BAP Health Outcomes Research

Pg. Ind. Espíritu Santo, parcela $37,1^{\circ} \mathrm{B}$

33010 Oviedo

E-mail: pablo@baphealth.com

Tel.: 985793704

Información artículo: Original - Incontinencia urinaria

Trabajo recibido: noviembre 2008

Trabajo aceptado: diciembre 2008 